\title{
A Portal to Salutogenesis in Languages of the World
}

\author{
Bengt Lindström
}

\section{Introduction}

Several considerations have triggered this Section of the book:

- First, Antonovsky claimed his key concepts within the salutogenic framework were universal, meaning SOC and GRRs could be studied in any society. The empirical evidence on SOC so far seems to support this with few exceptions.

- Second, the early critics of Antonovsky pointed out that the research mainly was concentrated around his own research team and some of his Scandinavian friends. This has never been true; we have researched on salutogenesis in all continents of the World and translations of the SOC instrument into at least 49 languages.

- Third, most scientific literature is today presented in English, meaning research in other main language groups does not appear in most literature searches. This leaves much cultural nuance outside the mainstream salutogenesis literature.

- Finally, it is about networking. Young researchers who plan to start studies in their countries and language groups will have great benefit of getting in contact with native researchers, enabling discussions and establishing national research teams and networks.

This section thus serves as a portal to salutogenesis writing of scholars working in languages other than English. There are already extensive language group networks such as the Spanish-Ibero American Network based at the

B. Lindström $(\square)$

NTNU Center for Health Promotion and Resources, Norwegian University of Science and Technology, Trondheim, Norway

e-mail: bengtblind@hotmail.com
Girona University, the French Canadian Network based in Montreal, and the German Network and other National networks.

Shortly before Antonovsky's death, he published the first international literature review on the SOC (Antonovsky, 1993). This included only a few publications because it was right after the introduction of salutogenesis in research. The second and much larger literature review published was the work of Lindstrom and Eriksson eventually leading to Eriksson's, 2007 Ph.D. dissertation 'Unravelling the Mystery of Salutogenesis,' and the establishment of the research database www.salutogenesis.fi later converted into www. salutogenesis.hv.se (Eriksson, 2007; Lindström \& Eriksson, 2010).

This chapter adds to the previous efforts, taking a new twist: salutogenesis scholars with mother tongue capability have summaries not the English language literature, but their own literatures in their own mother tongues. We do not, of course, cover all countries in which research on salutogenesis has been published in national languages. However, the following chapters do give a snapshot of salutogenesis research in many countries and most continents.

The rest of this chapter highlights some of the key perspectives in the literature on salutogenesis reviewed in the following chapters. Each language chapter has a concluding remark that partly is cited in this introduction. Most of the contributing scholars have conducted systematic reviews in national databases to compile the material for their text.

A general finding across the chapters of this section is that the second book by Antonovsky, Unravelling the Mystery of Health, has been translated to many of the languages included here. Also, the book that today serves as a primer of salutogenesis, the Hitchhikers Guide to Salutogenesis, is available in English, Spanish, and French (refs). It is also obvious that there is quite an extensive literature written in the old colonial languages: English of course, but also 
French, Spanish, and Portuguese, across national boundaries. This is also evident for literature in German. The same goes for the German speaking countries in Europe.

\section{Afrikaans, South African Union}

Although English is the main language in Afrikaans, South African Union (SAU) today, about seven million people speak Afrikaans, and a further 30 million people outside SAU speak the language. The SOC instruments have been translated into two other SAU languages beyond English and Afrikaans. The first study in Afrikaans was published in 1992. Most of the studies were cross-sectional descriptive studies, but there are a few evaluated interventions and conceptual studies. The main themes have been focused on psychology, industrial psychology, and sports. A total of 55 masters and Ph.D. studies addressing salutogenesis have been done in Afrikaans since 1992. Twenty-two of these have also been published in Afrikaans in scientific journals.

\section{Chinese}

The first study was undertaken in 2005 when the SOC 13 instrument was translated into Chinese. Most of the salutogenic literature in Chinese takes an empirical quantitative approach, measuring SOC and analyzing its relationship with other factors of interest. Most of the research just applies SOC sense of coherence as a tool without much consideration of the whole salutogenic model of health.

\section{Danish}

The Danish language literature on salutogenesis is rather limited, mainly focusing on quantitative approaches including population studies. Some of the first work written in Danish stems from work by Hollnagel and Malterud (2000) from the mid-late 1990s. They became international portal figures in the development of qualitative approaches to medicine. They suggested salutogenesis as a relevant foundation for talking with patients in general practice about risks and health resources. Some textbooks that reach into special areas like mental health and neuropedagogics underline the use of the salutogenic model in a practical and broader perspective. In general the majority of the Danish publications use salutogenesis as a theoretical framework from which various professions can work.

\section{Dutch}

There are at least four research groups in The Netherlands which carry out research in the area of salutogenesis; however most publish primarily in English. A special Dutch feature is salutogenesis-inspired research on sustainable agriculture, nutrition, and health. In the Dutch language literature, salutogenesis is mainly conceptualized in terms of the SOC, and one of its elements: meaningfulness. Researchers have used different definitions of the SOC, including integrative ability, self-curing ability, sense or feeling of coherence, awareness of coherence, world view, control over life, strengths, resilience, and empowerment. Remarkably, generalized resistance resources are hardly mentioned in the Dutch language literature.

\section{Finnish}

There has always been a tradition in Finland to publish scientific literature in the national language, also mirrored in the number of Ph.D. theses produced in Finnish. Research on salutogenesis started in the 1980s in Finland, rather early compared to other countries. Since the start, salutogenic research expanded into most health-related fields in Finland. Salutogenesis has often been used as one conceptual basis along with other theoretical foundations and concepts: health-related quality of life, coping, social support, selfesteem, and resources. A special Finnish feature is the focus on the relationship between the concepts of life control and sense of coherence.

\section{French}

The SOC-13 and SOC-29 were translated and validated in French in 2001. French language literature expands into most areas of salutogenic research including psychology, health promotion, management, and design. Salutogenesis has moved from an original concept, to a well-established theory, towards a unifying orientation, which finally unlocked a new field of research worldwide (i.e., positive approaches to health). French-speaking countries slowly, but surely, use the salutogenic road to create health.

\section{German}

Literature on salutogenesis in the German language is extensive covering two nations; unfortunately Austria was not able to present a review here. In Germany, a special feature is the interest within sociology to critically discuss the 
theoretical foundation of the salutogenic approach, mainly focused on the paradigmatic potential of the salutogenic approach vis-a-vis pathogenesis. In one of the German research groups, there is a strong emphasis on psychosomatic health and its practical implementations. In Switzerland, the number of studies of the SOC or other aspects of salutogenic theory is high, considering the fact that salutogenesis is virtually non-existent in academic considerations of health. However, there seem to be even more Master and Bachelor's theses related to salutogenic theory than formal research projects. This suggests that the interest of the coming academic generation in salutogenesis is strong-stronger perhaps than the interest shown so far by senior Swiss researchers.

\section{Hebrew}

Israel is the country where Antonovsky developed his salutogenic approach to health. Literature in Hebrew is extensive and broad-based, dating back to 1983. However, Antonovsky himself mainly published in English, eager to reach the international academic audience. In Hebrew, there were 175 titles found that focused on the sense of coherence and the salutogenic model up to 2014. Contentwise, the literature covers a broad approach to salutogenesis. The search for deeper understanding of the SOC at the personal and collective levels is of central importance, and maybe even of existential significance in the context of the Israeli reality. Exchanging the pathogenic questions about the emotional and physical cost of life in the stormy Israeli reality informs questions about resources that enable people in Israel to move towards growth and health. This can be the reason to move towards positive change in the social discourse in Israel, as well as strengthen the hope, durability, and adaptive ability of the residents of Israel. Another direction of research in the conflictual Israeli reality is the study of sense of the SOC in a more interdisciplinary way than in other countries, including studies on intergroup relationships and openness towards the "other."

\section{Italian}

The Italian language research on salutogenesis was initiated through the participation of Sardinia in the European Training Consortium (ETC) in 1993. Since then, a network of Italian academics has been involved in such research.

\section{Japanese}

The research on salutogenesis and the SOC in Japan started in 1996. Between 1997 and 2001 there were translations of Antonovsky's work and the creation of Japanese language versions of the SOC-29 and SOC-13. Today, academic literature in Japanese is impressive and extensive, covering many perspectives. Approximately 300 papers have been published in the last 11 years. The chapter here includes an account of the many scholars involved and a very interesting, detailed account of what goes on in salutogenic research in Japan.

\section{Norwegian}

One of the main research environments for salutogenesis in Norway is the Department of Health Promotion and Development at the University of Bergen, dedicated to health promotion research and teaching since 1986-however with most publications in English. There is, however, a long tradition of salutogenic research in Norway including all major Universities today and covering most aspects of this research area. An interesting trait is the strong emphasis on qualitative approachesoutnumbering quantitative approaches. This chapter gives a deep insight into the strong and many faceted nature of the Norwegian salutogenic literature. The Hitchhikers Guide to Salutogenesis was also translated into Norwegian in 2015.

\section{Polish}

Polish academics and practitioners got acquainted with the theory of salutogenesis in the 1980s, rather early compared to many other countries. This was quite likely stimulated by international conferences in psychology and bilateral research meetings. Publications in Polish language, reviewed in this chapter, are either theoretical analyses or research reports. In the former, salutogenesis is applied in a conceptual way, discussed in a variety of contexts, and related to other theoretical concepts. In the latter, the quantitative empirical approach is used and SOC is measured with validated tools. Findings of such studies provide data on correlates of the SOC and its relationship to many aspects of health and behavior. 


\section{Portuguese}

Salutogenesis was introduced to Portugal in 1994 through a visit of Antonovsky. The validation of the SOC instruments was undertaken between 1997 and 1999. The chapter on Portuguese salutogenesis literature gives a very detailed account. Struggling with concepts, their translation, and meaning in a different cultural setting than that of Antonovsky, continues today to be a challenge. New perspectives have emerged in this literature, like using the SOC framework to address changes in life styles like smoking cessation or brucellosis prevention. Addressing children's well-being and focusing on mental health promotion are areas where salutogenesis in association with other approaches can help achieve the goal of promoting health and favoring well-being.

\section{Spanish}

The scientific literature on salutogenesis that has been published in Spanish since the year 2000 has been reviewed, out of which 58 scientific articles, 5 books, and 8 doctoral theses have been identified. In just a few years, the influence of the salutogenic model has been growing, both in the area of research and in public health and health promotion strategies and policies, in Spain and in Latin America. The Network of Spanish Speaking Researchers on Salutogenesis has been very influential in the development of academic research in Spanish and Catalan. Today, the Spanishspeaking academic scene is many faceted and lively, as presented in this chapter.

\section{Swedish}

Antonovsky spent a Sabbatical in Lund, Sweden (1987-1988) and initiated salutogenic research both in Sweden and Scandinavia through his contacts at the Nordic School of Public Health in Gothenburg. He later became an
Honorary Doctor at the Nordic School (1993). Today salutogenesis has a productive, solid, and geographically broad-based foundation in Sweden. Many scholars and practitioners in different contexts are familiar and implement the salutogenic approach in research. The review for this chapter revealed that there are nowadays many handbooks and textbooks in Swedish on how to use the concept for studies in universities and colleges and in everyday practice. In line with this, a specific resource center on salutogenesis was established in 2011 at the University West in Trollhättan, aiming at support education on health and well-being, and conduct research on salutogenesis, and serving as a partner in collaboration and cooperation with the local community (www.salutogenesis.hv.se).

Open Access This chapter is distributed under the terms of the Creative Commons Attribution-Noncommercial 2.5 License (http:// creativecommons.org/licenses/by-nc/2.5/) which permits any noncommercial use, distribution, and reproduction in any medium, provided the original author(s) and source are credited.

The images or other third party material in this chapter are included in the work's Creative Commons license, unless indicated otherwise in the credit line; if such material is not included in the work's Creative Commons license and the respective action is not permitted by statutory regulation, users will need to obtain permission from the license holder to duplicate, adapt or reproduce the material.

\section{References}

Antonovsky, A. (1993). The structure and properties of the sense of coherence scale. Social Science \& Medicine, 36(6), 725-733.

Eriksson, M. (2007). Unravelling the mystery of health. Academic Thesis, Folkhalsan Research Center Report 1, Helsinki.

Hollnagel, H., \& Malterud, K. (2000). From risk factors to health resources. Medicine, Health Care, and Philosophy, 3, 257-274.

Lindström, B., \& Eriksson, M. (2010). Hitchhikers guide to salutogenesis. Folkhalsan Research Center Report 2, Helsinki.

Lindström, B., \& Eriksson, M. (2012a). Guia del Autoestopista Salutogenico, Girona Documenta Universitaria, Universita Girona, Spanish version in print and e-book.

Lindström, B., \& Eriksson, M. (2012b). La salutogene'se, petit guide pour promovoir la santé.

Lindström, B., \& Eriksson, M. (2015). Haikerens guide til salutogenese. Oslo: Gyldendal. 